\title{
Multi-channel terahertz grating spectrometer with quantum-cascade laser and microbolometer array
}

\author{
R. Eichholz, ${ }^{1}$ H. Richter, ${ }^{1}$ S. G. Pavlov, ${ }^{1}$ M. Wienold, ${ }^{2}$ L. Schrottke,${ }^{2}$ R. Hey, ${ }^{2}$ H. T. Grahn, ${ }^{2}$ \\ and H.-W. Hübers ${ }^{1,3, a)}$ \\ ${ }^{1}$ Institute of Planetary Research, German Aerospace Center (DLR), Rutherfordstr. 2, 12489 Berlin, Germany \\ ${ }^{2}$ Paul-Drude-Institut für Festkörperelektronik, Hausvogteiplatz 5-7, 10117 Berlin, Germany \\ ${ }^{3}$ Institut für Optik und Atomare Physik, Technische Universität Berlin, Hardenbergstraße 36, 10623 Berlin, \\ Germany
}

(Received 14 July 2011; accepted 13 September 2011; published online 6 October 2011)

\begin{abstract}
We report on a terahertz absorption spectrometer, which combines a grating monochromator, a quantum-cascade laser (QCL), and a microbolometer camera. The emission modes of the laser are spectrally resolved by the monochromator and imaged onto the camera. An absorption cell is placed between the QCL and the monochromator, and the absorption spectrum of methanol around 3.4 THz is measured by integrating simultaneously the signal of each of its Fabry-Pérot modes as a function of the laser driving current. The frequency coverage of the spectrometer is about $20 \mathrm{GHz}$. (C) 2011 American Institute of Physics. [doi:10.1063/1.3645635]
\end{abstract}

Many physical phenomena have characteristic energies, which correspond to terahertz $(\mathrm{THz})$ frequencies. Examples are rotational transitions of molecules, energy levels of shallow impurities in semiconductors, phononic spectra of solids, or the energy gaps of certain superconductors. A lot of basic research is devoted to investigate these phenomena. Also applied research is rapidly growing. Examples are security research, where the detection of explosives with characteristic $\mathrm{THz}$ spectra is a current topic, ${ }^{1,2}$ environmental research, for example, the detection of chemicals based on their rotational fingerprint spectra, ${ }^{3}$ or spectroscopy of biomedical samples such as skin or biomolecules. ${ }^{4,5}$

In the visible and infrared regions of the electromagnetic spectrum, broadband grating spectroscopy is a widely used technique. It was stimulated by the development of linear and matrix detector arrays. With such a multi-pixel detector, each pixel acts as a slit, and each spectral element is imaged onto one pixel. This allows for the simultaneous measurement of a broad spectral range. In addition, it facilitated the development of grating spectrometers without moving parts, because the grating does not need to be rotated. State-of-theart grating spectrometers in $\mathrm{THz}$ astronomy utilize arrays with several hundred photoconductive detectors cooled by liquid helium. The spectral information is dispersed across one dimension of the array, while the spatial information is contained in the other. An example is the photoconductor array camera and spectrometer (PACS) on board of ESA's Herschel space observatory. ${ }^{6}$ In addition, time domain spectrometers without moving parts have been realized using asynchronous optical sampling. ${ }^{7}$

$\mathrm{THz}$ quantum-cascade lasers (QCLs) are very attractive radiation sources for laser-based absorption spectroscopy. First, their emission is narrow in frequency (less than $20 \mathrm{kHz}$ ), which allows for a spectroscopy with very high resolution. Second, QCLs exhibit high output powers up to several tens of $\mathrm{mW}$, which allows for sensitive spectrometers, because the

\footnotetext{
a) Author to whom correspondence should be addressed. Electronic mail: heinz-wilhelm.huebers@dlr.de.
}

minimum detectable change of absorption depends on the power per bandwidth of the source. ${ }^{8,9}$ Since QCLs exhibit high output powers and narrow emission lines, their spectral brightness is very high. A third advantage is their broad gain medium, which in principle allows for a bandwidth of several $100 \mathrm{GHz}$ to be covered by a single laser. So far, highresolution molecular spectroscopy has been demonstrated only for a QCL with a distributed-feedback grating or for a QCL as a local oscillator in a heterodyne receiver. ${ }^{10,11}$ In both cases, the bandwidth covered by the spectrometer is only a few GHz. In principle, one can use the whole gain medium, if the QCL is integrated into an external cavity. This has been already demonstrated, and continuous, mode-hopping-free tuning was obtained over about $12 \mathrm{GHz} .^{12}$ In this letter, we report on a $\mathrm{THz}$ spectrometer, which takes advantage of the multi-mode emission of a QCL with a Fabry-Pérot resonator. The modes are spectrally resolved by a monochromator and imaged onto a microbolometer array.

The setup of the spectrometer is shown in Fig. 1. The main components are the QCL, a 27-cm long absorption cell, a grating monochromator (Acton SP2300), and an infrared camera (InfraTec GmbH VarioCAM). The potential of a microbolometer camera for $\mathrm{THz}$ imaging was pointed out in Ref. 13. The QCL uses intersubband transitions resonant to the longitudinal optical phonon for efficient carrier injection into the upper laser level. ${ }^{14}$ It has a 1.16 -mm long FabryPérot cavity and a single-plasmon (SP) waveguide. The QCL is mounted in a compact, air-cooled cryocooler (Ricor K535). ${ }^{15}$ The DC driving current for the QCL as well as an additional small sinusoidal current modulation are supplied by a battery-driven current source. The beam of the QCL is focused with a lens made from polymethylpentene (PMP, trade mark TPX) onto the entrance slit of the monochromator. The absorption cell is between the lens and the monochromator. It has two 1-mm-thick, high-density polyethylene windows, which are tilted in order to minimize standing waves. The pressure inside the cell is measured with a capacitance manometer. The monochromator is a Czerny-Turner type one with a blazed grating ( 8 lines $/ \mathrm{mm}$, blazed for 112 


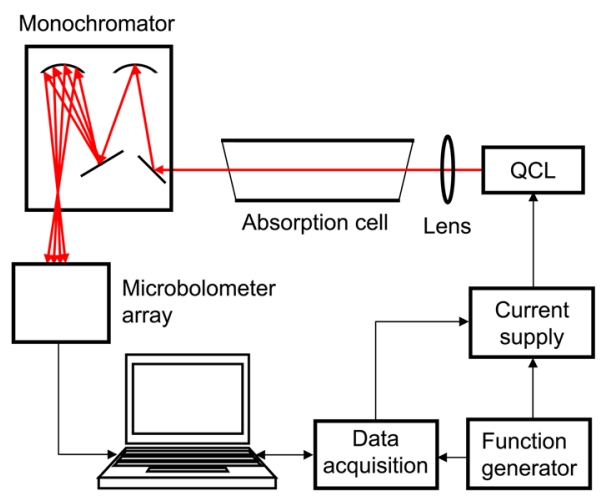

FIG. 1. (Color online) Experimental set-up of the spectrometer.

$\mu \mathrm{m})$. The entrance slit of the monochromator is $3 \mathrm{~mm}$ wide. The exit slit is completely opened (17 mm width). This allows for the simultaneous imaging of several laser modes without any additional optical element between the monochromator and the camera, which is placed behind the output. In this configuration, the spectral resolution is approximately $10 \mathrm{GHz}$. The camera is equipped with an objective lens made from germanium and a microbolometer array with $640 \times 480$ pixels $\left(16 \times 12 \mathrm{~mm}^{2}\right.$, pixel pitch $\left.25 \mu \mathrm{m}\right)$ made from amorphous silicon. The array is encapsulated in a vacuum housing with a germanium window. The camera is optimized for mid-infrared wavelengths $(8-14 \mu \mathrm{m})$. In order to use it at $\mathrm{THz}$ frequencies, the germanium lens in front of the camera was removed. The $50-\mathrm{Hz}$ frame rate of the camera is determined by the data integration time of 8 and $12 \mathrm{~ms}$ for subsequent data handling.

The emission spectrum of the QCL measured with the microbolometer array at the exit of the monochromator is shown in Fig. 2 for three different driving currents and temperatures. In total, four longitudinal modes (I-IV) separated by approximately $31 \mathrm{GHz}$ can be detected by the microbolometer array. Frequency calibration was done with a Golay detector and the monochromator operating in its standard mode. The absolute power of each mode and the power distribution between the modes depend on the driving current and the laser temperature. The total power increases and modes with higher frequencies become more intense at higher driving currents. The power of the modes was calibrated with respect to a Golay cell detector. At $450 \mathrm{~mA}$, it is about $0.1 \mathrm{~mW}$, while it amounts to about $0.3 \mathrm{~mW}$ at $550 \mathrm{~mA}$ and $650 \mathrm{~mA}$ for the strongest mode at the exit of the monochromator.

In order to evaluate the spectroscopic capabilities of this set-up, the gas cell was filled with methanol $\left(\mathrm{CH}_{3} \mathrm{OH}\right)$ at $1 \mathrm{hPa}$ pressure. At this pressure, the full width at half maximum (FWHM) of the absorption lines is approximately $25 \mathrm{MHz} .{ }^{10,16}$ The power transmitted through the cell was detected with the microbolometer array as a function of the driving current of the QCL. All laser modes are integrated simultaneously by the microbolometer array before readout. The integration time of the lock-in amplifier was $500 \mathrm{~ms}$ per current step of $0.65 \mathrm{~mA}$. An AC current with a modulation frequency of $5 \mathrm{~Hz}$ (ten times less than the frame rate of the camera) and an amplitude of $1.7 \mathrm{~mA}$ was superimposed onto the DC current. A LABVIEW@ program with implemented lock-

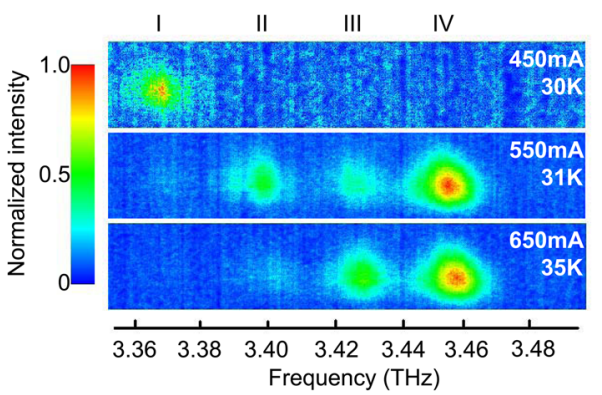

FIG. 2. (Color online) Emission modes I, II, III, and IV of the QCL imaged with a microbolometer array at the output of the monochromator. The QCL operating conditions are given on the right-hand side. Each image contains $100 \times 500$ pixels of the full image of the microbolometer array width. The output power of each image is normalized to the maximum value.

in amplifier was used. The signal of each mode was integrated by the software, and the resulting value was detected by the lock-in amplifier for each mode separately and simultaneously. An example of the spectra is shown in Fig. 3. In the spectral range of each laser mode, at least two absorption lines of $\mathrm{CH}_{3} \mathrm{OH}$ are present. In Fig. 3, the signal-to-noise ratio (SNR) is given for all absorption lines. The root-meansquare noise is the same in all spectra indicating that it is limited by the microbolometer array. In addition, the SNR depends on the power of the laser mode and the absorption intensity of the transition. In order to estimate the influence of the laser power, one may look at the lines with the best SNR in each mode spectrum. These lines have approximately the same absorption intensity (within a factor of 1.5 (Ref. 17)). The SNR scales approximately with the power of the laser modes at these frequencies $(0.1,0.3,<0.1$, and $0.3 \mathrm{~mW}$ from mode I to mode IV). In particular, the poor SNR in the spectrum of mode 3 is caused by the rather small available laser power.

The frequency calibration was performed by comparing the detected positions with published data of $\mathrm{CH}_{3} \mathrm{OH}$ absorption lines measured with a Fourier transform spectrometer (FTS). ${ }^{17}$ The frequency tuning of the four modes as derived from a comparison with the FTS data is shown in Fig. 4. It varies between 12 and $31 \mathrm{MHz} / \mathrm{mA}$. Note that the linear fit of the tuning which is used in Fig. 4 is only a rough estimate, because of the limited number of absorption lines per mode. The observed tuning characteristic is caused by a change of the effective refractive index due to temperature and current changes. It appears that for a higher emission frequency of the QCL the tuning rate becomes larger. This is explained by a different change of the effective refractive index below and above the gain maximum of the QCL.

The measured FWHM of the absorption lines varies between about $30 \mathrm{MHz}$ for the low-frequency lines at $3.363 \mathrm{THz}$ and about $100 \mathrm{MHz}$ for the high-frequency lines at $3.459 \mathrm{THz}$. At $1 \mathrm{hPa}$, the linewidths of the rotational transitions of $\mathrm{CH}_{3} \mathrm{OH}$ are dominated by pressure broadening and are approximately $25 \mathrm{MHz}{ }^{10,16}$ The Doppler broadening of $\mathrm{CH}_{3} \mathrm{OH}$ at $300 \mathrm{~K}$ is $5.5 \mathrm{MHz}$. Additional instrumental broadening effects are caused by frequency modulation, which is about $20 \mathrm{MHz}$ at $3.363 \mathrm{THz}$, and the finite steps of the frequency scan of about $8 \mathrm{MHz}$. At $3.459 \mathrm{THz}$, these values become about 53 and about $20 \mathrm{MHz}$, respectively. These 


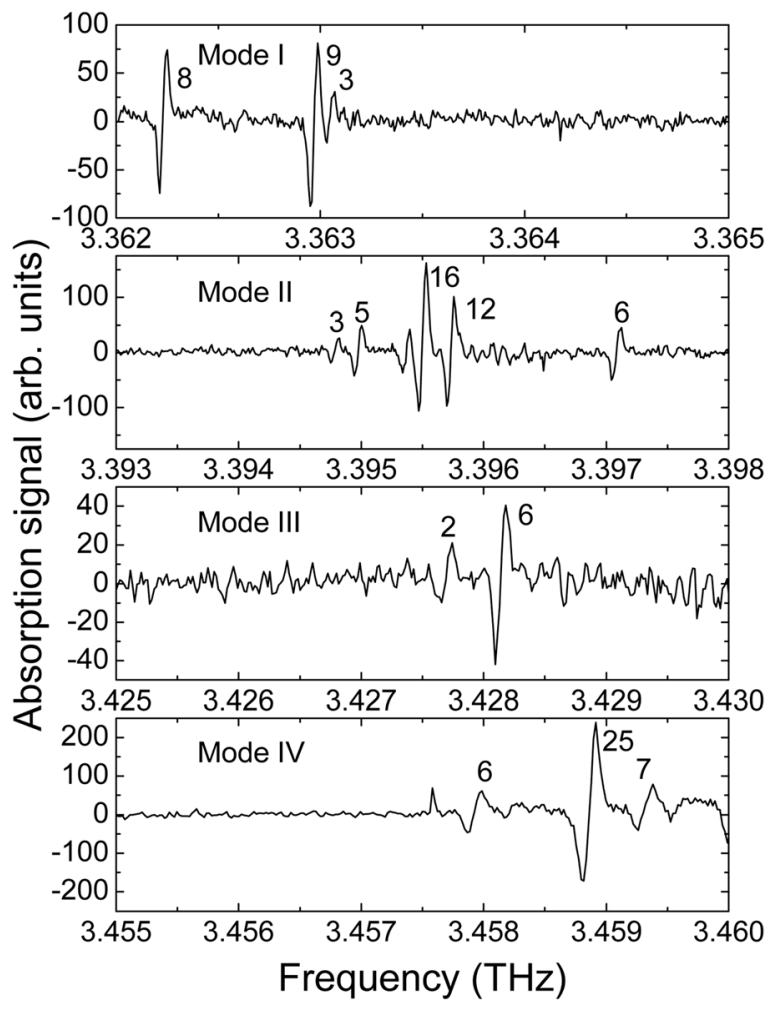

FIG. 3. Spectra of $\mathrm{CH}_{3} \mathrm{OH}$ at $1 \mathrm{hPa}$ obtained from mode I, II, III, and IV. All modes were measured simultaneously. The numbers given next to each absorption line are the SNR.

additional contributions lead to the observed linewidths. The frequency coverage is about $5 \mathrm{GHz}$ per mode, yielding a total instantaneous frequency coverage of about $20 \mathrm{GHz}$.

The experiments demonstrate that a broadband $\mathrm{THz}$ spectrometer with no moving parts based on a QCL is feasible. Several improvements can be envisaged. First of all, a more sensitive detector array will improve the SNR. Second, the absolute frequency accuracy is limited by the $\mathrm{CH}_{3} \mathrm{OH}$ reference spectrum measured with an FTS. The positions of the lines are reproducible within $5 \mathrm{MHz}$. By using more precise reference data, it is possible to significantly improve the accuracy. For example, the rotational transitions of $\mathrm{CO}$ are known with an accuracy of $\pm 20 \mathrm{kHz} .{ }^{18}$ Also a Fabry-Pérot interferometer might be used to allow for a more precise frequency interpolation between the frequencies of the two molecular reference lines. It is advantageous to measure both the molecule under investigation and the reference spectrum simultaneously, because the precise sub-MHz repetition of the frequency in two subsequent frequency scans is difficult to achieve due to thermal hysteresis and the sensitive dependence of the frequency on the laser temperature. Another improvement is associated with the frequency coverage. With dedicated optics, the emission of a QCL can be focused to a spot of $1 \mathrm{~mm}$ diameter. To minimize crosstalk between two modes on the array, they could be imaged at a distance of $2 \mathrm{~mm}$. This leads to eight modes per row for an array as used in the present camera. In an echelle-type configuration of the $\mathrm{THz}$ spectrometer, six rows can be stacked on top of each other. This leads to a total number of 48 laser modes on the array. If each mode can be fine tuned by about $10 \mathrm{GHz}$,

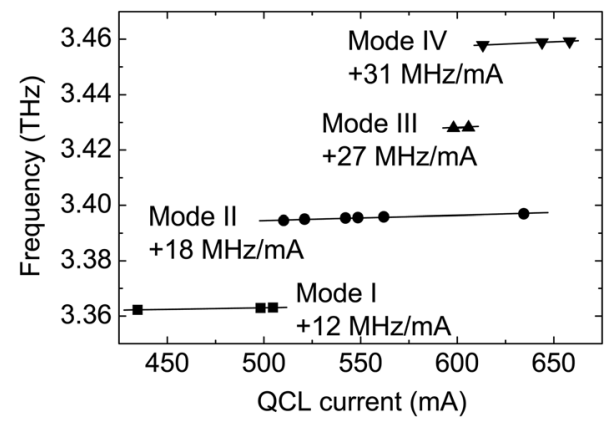

FIG. 4. Frequency tuning of the emission modes I, II, III, and IV of the QCL versus driving current. The tuning rates given next to each curve are derived from the tabulated frequencies of $\mathrm{CH}_{3} \mathrm{OH}$ absorption lines. The straight lines are linear fits to the data points.

the total coverage of such a spectrometer is approximately $0.5 \mathrm{THz}$. Obviously, this requires an optimized QCL with an adequate mode spacing and frequency tuning rate as well as a broad gain medium. Using this approach, the realization of broadband $\mathrm{cw} \mathrm{THz}$ spectrometers with no moving parts appears to be feasible.

This work was supported by the European Commission through the ProFIT program of the Investitionsbank Berlin. R.E. acknowledges support by the Helmholtz Research School on Security Technologies.

${ }^{1}$ Y. C. Shen, T. Lo, P. F. Taday, B. E. Cole, W. R. Tribe, and M. C. Kemp, Appl. Phys. Lett. 86, 241116 (2005).

${ }^{2}$ M. Ortolani, J. S. Lee, U. Schade, and H.-W. Hübers, Appl. Phys. Lett. 93, 081906 (2008).

${ }^{3}$ I. R. Medvedev, C. F. Neese, G. M. Plummer, and F. C. De Lucia, Opt. Lett. 35, 1533 (2010).

${ }^{4}$ R. M. Woodward, B. E. Cole, V. P. Walker, R. J. Pye, D. D. Arnone, E. H. Linfield, and M. Pepper, Phys. Med. Biol. 47, 3853 (2002).

${ }^{5}$ U. Heugen, G. Schwaab, E. Bründermann, M. Heyden, X. Yu, D. M. Leitner, and M. Havenith, Proc. Natl. Acad. Sci. U.S.A. 103, 12301 (2006).

${ }^{6}$ A. Poglitsch, C. Waelkens, N. Geis, H. Feuchtgruber, B. Vandenbussche, L. Rodriguez, O. Krause, E. Renotte, C. van Hoof, P. Saraceno et al., Astron. Astrophys. 518, L2 (2010).

${ }^{7}$ A. Bartels, R. Cerna, C. Kistner, A. Thoma, F. Hudert, C. Janke, and T. Dekorsy, Rev. Sci. Instrum. 78, 035107 (2007).

${ }^{8}$ B. S. Williams, Nature Photon. 1, 517 (2007).

${ }^{9}$ H.-W. Hübers, M. F. Kimmitt, N. Hiromoto, and E. Bründermann, IEEE Trans. Terahertz Sci. Technol. 1, 321 (2011).

${ }^{10}$ H.-W. Hübers, S. G. Pavlov, H. Richter, A. D. Semenov, L. Mahler, A. Tredicucci, H. E. Beere, and D. A. Ritchie, Appl. Phys. Lett. 89, 061115, (2006).

${ }^{11}$ Y. Ren, J. N. Hovenier, R. Higgins, J. R. Gao, T. M. Klapwijk, S. C. Shi, B. Klein, T.-Y. Kao, Q. Hu, and J. L. Reno, Appl. Phys. Lett. 98, 231109 (2011).

${ }^{12}$ J. Xu, J. M. Hensley, D. B. Fenner, R. P. Green, L. Mahler, A. Tredicucci, M. G. Allen, F. Beltram, H. E. Beere, and D. A. Ritchie, Appl. Phys. Lett. 91, 121104 (2007).

${ }^{13}$ A. W. M. Lee and Q. Hu, Opt. Lett. 30, 2563 (2005).

${ }^{14}$ M. Wienold, L. Schrottke, M. Giehler, R. Hey, W. Anders, and H. T. Grahn, Electron. Lett. 45, 1030 (2009).

${ }^{15}$ H. Richter, M. Greiner-Bär, S. G. Pavlov, A. D. Semenov, M. Wienold, L. Schrottke, M. Giehler, R. Hey, H. T. Grahn, and H.-W. Hübers, Opt. Express 18, 10177 (2010).

${ }^{16}$ P. Minguzzi, M. Tonelli, G. Carrara, and A. Di Lieto, J. Mol. Spectrosc. 109, 395 (1985).

${ }^{17}$ G. Moruzzi, P. Riminucci, F. Strumia, B. Carli, M. Parlotti, R. M. Lees, I. Mukhopadhyay, J. W. C. Johns, B. P. Winnewisser, and M. Winnewisser, J. Mol. Spectrosc. 144, 139 (1990).

${ }^{18}$ T. D. Varberg and K. M. Evenson, Astrophys. J. 385, 763 (1992). 